\title{
Genome Size of Twenty Wild Species of Oryza Determined by Flow Cytometric and Chromosome Analyses
}

\author{
Toshie Miyabayashi ${ }^{1)}$, Ken-Ichi Nonomura ${ }^{1,2)}$, Hiroko Morishima ${ }^{3)}$ and Nori Kurata ${ }^{* 1,2)}$ \\ 1) National Institute of Genetics, Yata 1111, Mishima, Shizuoka 411-8540, Japan \\ 2) Graduate University for Advanced Studies/Sokendai, Yata 1111, Mishima, Shizuoka 411-8540, Japan \\ 3) Saiwai-cho 15-2, Hiratsuka, Kanagawa 254-0804, Japan
}

\begin{abstract}
The National Institute of Genetics (NIG), Mishima, Japan, maintains 1,725 accessions of 20 wild species of the genus Oryza, which have been collected from tropical and subtropical areas all over the world and covering all species in the genus except for $O$. schlechteri Pilger. A core collection of 282 accessions was chosen to represent the genetic spectrum of the whole NIG collection. The genome sizes of 39 accessions of Oryza core collection, covering all 20 species and 9 genome types, were measured based on both flow cytometry and microscopic observation. Most accessions showed good correspondence with respect to genome size within the same species and genome type, but showed significant differences between different genome types. In contrast to other species, however, CC, CCDD and HHJJ species showed significant variation within the same genome type. The total somatic chromosome length and ploidy level were also investigated for several accessions, and they correlated well with the DNA content. In this study, a simple flow cytometric analysis provided reliable results for genome size evaluation, and in addition, the genome size of five species was newly determined. Thus the DNA content, which is the simplest reliable indicator of genome variability, should be a good means of monitoring for evaluation and identification of Oryza germplasm.
\end{abstract}

Key Words: Oryza, genetic diversity, flow cytometry, DNA content, genome size, chromosome, species differentiation.

\section{Introduction}

The genus Oryza consists of more than 20 wild and two cultivated species, including eight tetraploids in addition to diploids (Vaughan and Morishima 2003). The cultivated species, $O$. sativa L. and $O$. glaberrima Steud., and five wild species closely related to the cultivars share the AA genome. Agronomically useful traits of wild AA species can be introduced into cultivars by a conventional hybridization method because these genes are readily transferred, and many interspecific hybrids and their derivatives have been developed to identify useful genes including quantitative trait loci (reviewed in Khush 1997). On the other hand, the fifteen wild species with genomes other than AA are designated as BB, $\mathrm{CC}, \mathrm{EE}, \mathrm{FF}$ and GG diploids, and $\mathrm{BBCC}, \mathrm{CCDD}$ and HHJJ tetraploids (Vaughan and Morishima 2003), according to the genomic homology judged by the degree of meiotic chromosome pairing in interspecific hybrids. These remote species carry many resistance genes for insects and diseases (Brar and Khush 1997), but are difficult to use in rice breeding, due to limited recombination and low affinity of their chromosomes to the AA chromosomes of rice during meiosis.

Communicated by Darshan S. Brar

Received September 4, 2006. Accepted November 3, 2006.

*Corresponding author (e-mail: nkurata@lab.nig.ac.jp)
Although several researchers have tried to introduce agronomically useful traits of remote species into cultivated rice (Brar and Khush 1997, Multani et al. 2003, Jena et al. 2006), rice breeding using hybridization between distant genomes is still far from routine. To understand the genetic basis of various reproductive barriers in interspecific hybrids, reproductive traits and genes have also been studied in rice (reviewed in Kurata et al. 2005). The evaluation of Oryza species from many standpoints is important to provide useful germplasm to researchers and breeders.

The National Institute of Genetics (NIG), Mishima, Japan, maintains 1,725 accessions of wild Oryza species collected from tropical and subtropical areas of the world. We set up a core collection, a subset of all accessions chosen to represent the genetic spectrum of the whole NIG collection according to the concept proposed by Frankel (1984). The NIG core collection contains 20 wild species, which cover all Oryza species except for $O$. schlechteri Pilger, with 9 genome types. Several accessions were selected from each species to represent the whole range of variation of respective species, considering all available information about original habitat and phenotypic and genetic data. The NIG core collection is divided into three ranks of 44 (rank 1), 65 (rank 2) and 173 accessions (rank 3 ) to meet variable aims of users. Various information on all accessions, especially the accessions included in the core collection, is available online from 
the Oryzabase web site (http://www.shigen.nig.ac.jp/rice/ oryzabase/top/top.jsp). Duplicate stocks of most of these accessions have been maintained at the International Rice Research Institute (IRRI) since a few decades ago. Recently, some of these wild species have been used to construct bacterial artificial chromosome libraries (Ammiraju et al. 2006). In this study, 39 accessions of 20 species of the NIG core collection were used to determine variability in genome size among species and genome types. Flow cytometry of leaf nuclei and analysis of somatic chromosomes were carried out to examine the DNA content in several genome types and species. The simple flow cytometric and chromosome analyses proved useful for evaluation and classification of unknown or doubtful accessions of a given species.

\section{Materials and Methods}

\section{Plant material}

Thirty-nine accessions of the 20 wild Oryza species used in this study were derived from the core collection maintained by the NIG, Japan (http://www.shigen.nig.ac.jp/ rice/oryzabase/nbrpStrains/nig.jsp). According to conventional classification, O. punctata has two different types, a diploid BB type and a tetraploid BBCC type (Vaughan and Morishima 2003). In this study, these two types were treated as different species, $O$. punctata diplo. and O. punctata tetra. Of the 39 accessions used, 27 were chosen from rank 1, nine from rank 2 , and three from rank 3 . The species name, genome type, and accession number of all plant materials are listed in Table 1. Seed dormancy was broken by heating at $42^{\circ} \mathrm{C}$ for a week, then the seeds were dehusked and germinated on a wet sheet. Germinated seeds were transplanted into soil and grown in the field or in a greenhouse at around $30^{\circ} \mathrm{C}$ during the day and 20 to $24^{\circ} \mathrm{C}$ at night in Mishima, Shizuoka, Japan.

\section{Flow cytometry}

Flow cytometric procedures were performed on the ploidy analyzer PA system (Partec, USA) using the modified method described by Galbraith et al. (1983). Nuclei of Oryza species were isolated from about $5 \mathrm{~mm}^{2}$ of fresh adult leaves or young leaves at 30 to 60 days after germination. A leaf square was chopped into fine pieces with a razor blade in $400 \mu \mathrm{L}$ of extraction solution A (Partec) and placed at room temperature for $30 \mathrm{~min}$. The supernatant was filtered in one step using CellTrics filters with 50 and $20 \mu \mathrm{m}$ pore sizes (Partec), stained with 4',6-diamidino-2-phenylindole (DAPI) by adding $160 \mu \mathrm{L}$ of staining solution B (Partec) to the suspension, and placed at room temperature for $30 \mathrm{~min}$. Signals of DAPI-stained nuclei were processed with 400-440 $\mathrm{nm}$ excitation filter UG1 and $435 \mathrm{~nm}$ absorbance filter GG435 by the ploidy analyzer PA flow cytometer (all Partec). We calculated DNA content of wild species by comparison of mean values of fluorescent intensity to those of cv. Nipponbare (a standard variety). Standard measurements were performed after every two measurements of wild spe- cies. The difference between two neighboring standards was divided by 3 (D value), and measurements of the two samples of wild species between the standards were normalized by the $\mathrm{D}$ value. The value of $0.91 \mathrm{pg} / 2 \mathrm{C}$ was used as the DNA content of cv. Nipponbare as generally accepted (Ammiraju et al. 2006). About 2,000 nuclei were analyzed per sample, and for each accession, the measurement was repeated three times, with measurements on different days.

\section{Chromosome observation}

The flame-drying method described by Kurata et al. (1981) was applied for spreading mitotic chromosomes with slight modifications. Young roots from germinating seeds were fixed with a fixative ( $3: 1$ ethanol: acetic acid). Root tip tissue was put on a glass slide and treated with $4 \%$ cellulase Onozuka RS (Yakult Honsha, Japan), 2\% pectolyase Y-23 (Kikkoman, Japan), $1 \mathrm{mM}$ EDTA, and $75 \mathrm{mM} \mathrm{KCl,} \mathrm{pH} 4.2$ at $37^{\circ} \mathrm{C}$ for 30 to $35 \mathrm{~min}$. After rinsing with distilled water, the root tip was squashed in fixative and flame-dried on a glass slide. Chromosome spreads were stained with $4 \%$ Giemsa (Sigma, USA) in $33 \mathrm{mM}$ phosphate buffer ( $\mathrm{pH} 6.8$ ) for 20 min, then photographed using a BX50 light microscope with a DP50 CCD camera system (Olympus, Japan). The total length of prometaphase chromosomes was measured by Image-J 1.36b software (Abramoff et al. 2004).

\section{Results}

\section{Flow cytometry}

A single peak corresponding to the $2 \mathrm{C}$ level $\left(\mathrm{G}_{0}+\mathrm{G}_{1}\right.$ phase), but not to the $4 \mathrm{C}\left(\mathrm{G}_{2}+\mathrm{M}\right.$ phase) was found by flow cytometric analysis of nuclei isolated from rice leaves (Martinez et al. 1994). Thus, in this study, the 2C DNA content of leaf nuclei was compared for 39 accessions of 20 wild Oryza species (Table 1). The data obtained from previous works are also given for most of the strains used in this study. The results indicate that accessions belonging to the same species and same genome type seem to have similar genome sizes, while the genome size differed significantly among genome types (variance ratio $\mathrm{F}=43.24$ ). The average DNA contents were statistically compared among the nine different genome types using a multiple range test (Snedecor 1956) (Table 2). The FF genome of $O$. brachyantha showed the smallest DNA content in this study, whereas differences were not significant within the $\mathrm{AA}, \mathrm{BB}$ and $\mathrm{FF}$ genome types. The AA genome species and O. punctata diplo. (BB) exhibited similar DNA contents, and the DNA contents of $\mathrm{CC}$ species (O. eichingeri, O. officinalis and O. rhizomatis) were slightly larger than those of AA and BB species. The EE and GG genome species showed significantly larger DNA contents than the other diploid species, being similar to those of tetraploid species. The HHJJ genome, including $O$. longiglumis and $O$. ridleyi, showed the largest DNA contents. For most genome types, the DNA contents corresponded well to those of previous studies (Ammiraju et al. 2006, Uozu et al. 1997, Martinez et al. 1994) (Table 1). 
Table 1. Nuclear DNA content of Oryza species measured by flow cytometry

\begin{tabular}{|c|c|c|c|c|c|c|c|}
\hline \multirow{3}{*}{ Oryza species } & \multirow{2}{*}{\multicolumn{2}{|c|}{ Accession No. ${ }^{\text {a }}$ or variety name }} & \multirow{3}{*}{$\begin{array}{l}\text { Genome } \\
\text { type }\end{array}$} & \multirow{3}{*}{ Country } & \multicolumn{3}{|c|}{ DNA content } \\
\hline & & & & & \multirow{2}{*}{$\begin{array}{c}\text { This study } \\
\text { pg/2C } \pm \text { S.D. }\end{array}$} & \multicolumn{2}{|c|}{ Previous data } \\
\hline & NIG & IRRI & & & & $\mathrm{pg} / 2 \mathrm{C}$ & Reference $^{b}$ \\
\hline O. sativa & \multicolumn{2}{|c|}{ cv. Nipponbare } & AA & Japan & $0.91^{\mathrm{c}}$ & 0.91 & $1,2,3$ \\
\hline \multirow[t]{3}{*}{ O. rufipogon } & W1294 & 105568 & AA & Philippines & $0.91 \pm 0.06$ & & \\
\hline & W1866 & 104650 & AA & Thailand & $0.87 \pm 0.08$ & & \\
\hline & W0120 & 105491,101470 & AA & India & & $0.91,0.95$ & 1,2 \\
\hline \multirow[t]{2}{*}{ O. barthii } & W0698 & & $\mathrm{AA}$ & Guinea & $0.94 \pm 0.15$ & & \\
\hline & W1588 & 105608 & AA & Cameroun & $0.95 \pm 0.05$ & & \\
\hline \multirow[t]{3}{*}{ O. longistaminata } & W1413 & 101228 & $\mathrm{AA}$ & Sierra Leone & $0.93 \pm 0.08$ & & \\
\hline & W1508 & 105069,101435 & AA & Madagascar & $0.89 \pm 0.07$ & & \\
\hline & W0029 & & AA & India & & 0.81 & 2 \\
\hline \multirow[t]{3}{*}{ O. glumaepatula } & W1169 & & $\mathrm{AA}$ & Cuba & $0.98 \pm 0.03$ & & \\
\hline & W2199 & & AA & Brazil & $1.05 \pm 0.06$ & & \\
\hline & W1246 & & AA & Brazil & & 0.99 & 2 \\
\hline \multirow{2}{*}{ O. meridionalis } & W1625 & 104085 & $\mathrm{AA}$ & Australia & $0.88 \pm 0.07$ & 1.02 & 2 \\
\hline & W1635 & 104092 & $\mathrm{AA}$ & Australia & $0.90 \pm 0.04$ & & \\
\hline \multirow[t]{3}{*}{ O. punctata diplo. } & W1514 & 105690 & $\mathrm{BB}$ & Kenya & $0.86 \pm 0.17$ & 0.88 & 1 \\
\hline & W1590 & 104071 & BB & Cameroun & $0.85 \pm 0.08$ & & \\
\hline & W1582 & 104067 & $\mathrm{BB}$ & Chad & & 1.11 & 2 \\
\hline \multirow[t]{2}{*}{ O. punctata tetra. } & W1024 & 101409 & $\mathrm{BBCC}$ & Ghana & $2.02 \pm 0.04$ & & \\
\hline & W1408 & 105153,101329 & $\mathrm{BBCC}$ & Nigeria & $1.90 \pm 0.13$ & & \\
\hline \multirow[t]{3}{*}{ O. minuta } & $\mathrm{W} 1213^{\mathrm{d}}$ & 100174,100973 & $\mathrm{BBCC}$ & Philippines & $1.67 \pm 0.23$ & & \\
\hline & W1331 & 101132 & $\mathrm{BBCC}$ & Philippines & $1.92 \pm 0.08$ & & \\
\hline & W1342 & 101141 & $\mathrm{BBCC}$ & Philippines & & 2.33 & 3 \\
\hline \multirow[t]{3}{*}{ O. officinalis } & W0002 & 100896,100878 & $\mathrm{CC}$ & Thailand & $1.19 \pm 0.05$ & $1.35,1.45$ & 1,2 \\
\hline & W1830 & & $\mathrm{CC}$ & unknown & $1.36 \pm 0.20$ & & \\
\hline & & 101150 & $\mathrm{CC}$ & Malaysia & & 1.14 & 3 \\
\hline \multirow[t]{4}{*}{ O. eichingeri } & $\mathrm{W} 1525^{\mathrm{d}}$ & 101427,105181 & $\mathrm{CC}$ & Uganda & $1.64 \pm 0.08$ & & \\
\hline & $\mathrm{W} 1527^{\mathrm{d}}$ & 101429,105182 & $\mathrm{CC}$ & Uganda & $1.11 \pm 0.05$ & & \\
\hline & W1520 & 101422 & $\mathrm{CC}$ & Uganda & & 1.17 & 3 \\
\hline & W1521 & 101423 & $\mathrm{CC}$ & Uganda & & 1.47 & 2 \\
\hline O. rhizomatis & W1805 & 103410 & $\mathrm{CC}$ & Sri Lanka & $1.92 \pm 0.17$ & & \\
\hline O. alta & W0017 & 100888 & CCDD & Surinam & $1.68 \pm 0.33$ & & \\
\hline & W1147 & 105557 & CCDD & Surinam & $2.35 \pm 0.14$ & & \\
\hline & W1182 & 105143 & CCDD & Guyana & $2.04 \pm 0.06$ & 2.09 & 1 \\
\hline O. latifolia & W2200 & & CCDD & Brazil & $1.88 \pm 0.01$ & & \\
\hline & W1177 & 100965 & CCDD & Costa Rica & & 2.32 & 3 \\
\hline O. grandiglumis & W1194 & 105144 & CCDD & Brazil & $2.06 \pm 0.05$ & & \\
\hline & W2220 & & CCDD & Brazil & $2.10 \pm 0.18$ & & \\
\hline & W1189 & 101405 & CCDD & Brazil & & 1.99 & 3 \\
\hline O. australiensis & W0008 & 100882 & $\mathrm{EE}$ & Australia & $1.92 \pm 0.15$ & $2.00,1.99$ & 1,3 \\
\hline & W1628 & & $\mathrm{EE}$ & Australia & $1.93 \pm 0.05$ & & \\
\hline & W1538 & 105165 & $\mathrm{EE}$ & Australia & & 1.96 & 2 \\
\hline O. brachyantha & W1401 & 101232 & $\mathrm{FF}$ & Sierra Leone & $0.63 \pm 0.15$ & $0.75,0.72$ & 1,2 \\
\hline & W1407 & 105570,101236 & $\mathrm{FF}$ & Mali & $0.60 \pm 0.08$ & & \\
\hline O. granulata & W0003 & 100879 & GG & India & $2.29 \pm 0.25$ & & \\
\hline & W0067 & 102118 & GG & Thailand & $2.46 \pm 0.26$ & 1.83 & 1 \\
\hline O. meyeriana & W1348 & 104503 & GG & Malaysia & $2.40 \pm 0.24$ & & \\
\hline & W1356 & 104989 & GG & Malaysia & $2.27 \pm 0.21$ & & \\
\hline O. ridleyi & W0001 & 100821 & HНJJ & Thailand & $2.03 \pm 0.33$ & $2.66,1.93$ & 1,3 \\
\hline & W0604 & 105134 & HНJJ & Malaysia & $3.00 \pm 0.23$ & & \\
\hline O. longiglumis & W1224 & & HHJJ & New Guinea & $2.72 \pm 0.21$ & & \\
\hline & W1228 & & HНJJ & New Guinea & $2.91 \pm 0.15$ & & \\
\hline
\end{tabular}

a: In many cases, accession numbers are designated for duplicated stocks preserved both in the NIG and IRRI.

b: 1; Ammiraju et al. (2006), 2; Uozu et al. (1997), 3; Martinez et al. (1994).

c: DNA content of $O$. sativa $\mathrm{ssp}$. japonica $\mathrm{cv}$. Nipponbare $(0.91 \mathrm{pg} / 2 \mathrm{C})$ was used as a standard.

$\mathrm{d}$ : For these strains, ploidy level and species name designation at NIG are different from those at IRRI (see text). 
Table 2. Comparison of DNA contents among genome types of the genus Oryza

\begin{tabular}{|c|c|c|c|c|c|c|c|}
\hline \multirow{2}{*}{$\begin{array}{c}\text { Genome } \\
\text { type }\end{array}$} & \multicolumn{2}{|c|}{ DNA content $(\mathrm{pg} / 2 \mathrm{C})^{\mathrm{a}}$} & \multirow{2}{*}{\multicolumn{5}{|c|}{ Multiple range test ${ }^{\mathrm{b}}$}} \\
\hline & Mean & Range & & & & & \\
\hline $\mathrm{FF}$ & 0.61 & $0.60-0.63$ & $\mathrm{a}$ & & & & \\
\hline BB & 0.86 & $0.85-0.86$ & $\mathrm{a}$ & & & & \\
\hline AA & 0.93 & $0.87-1.05$ & $\mathrm{a}$ & $\mathrm{b}$ & & & \\
\hline $\mathrm{CC}$ & 1.39 & $1.11-1.92$ & & $\mathrm{~b}$ & $\mathrm{c}$ & & \\
\hline $\mathrm{BBCC}$ & 1.88 & $1.67-2.02$ & & & $\mathrm{c}$ & d & \\
\hline $\mathrm{EE}$ & 1.92 & $1.92-1.93$ & & & & $\mathrm{~d}$ & \\
\hline CCDD & 2.02 & $1.68-2.35$ & & & & $\mathrm{~d}$ & \\
\hline GG & 2.35 & $2.27-2.46$ & & & & $\mathrm{~d}$ & e \\
\hline HHJJ & 2.67 & $2.03-3.00$ & & & & & $\mathrm{e}$ \\
\hline
\end{tabular}

a. The mean of DNA contents was calculated on the basis of Table 1.

b: Any two means tagged by the same alphabet are not significantly different at $5 \%$ level.

However, in several species with a large genome size (O. granulata and $O$. ridleyi), the DNA contents were markedly different from those reported previously.

Variation in DNA content differed remarkably among genome types. In contrast to other genome types, the CC, CCDD and HHJJ species showed diverse genome sizes (Table 2). O. rhizomatis had a significantly larger DNA content than the other $\mathrm{CC}$ species (Table 1). The accession W0017 of O. alta and W2200 of O. latifolia, both carrying the CCDD genome, showed an extremely small DNA content compared to the other CCDD species (Table 1). The genome size of five species, $O$. barthii (AA), O. punctata tetra. (BBCC), O. rhizomatis (CC), O. meyeriana (GG) and O. longiglumis (HHJJ), was determined for the first time in this study (Table 1).

\section{Microscopic chromosome analysis}

It is difficult to judge the ploidy level only from flow cytometric results because the diploid EE and GG species had a larger DNA content than several tetraploids (Table 2). We determined chromosome numbers and total lengths in several CC and GG genome species (Table 3), since the species names of several accessions of the $\mathrm{CC}$ genome species have been assigned differently by the NIG and IRRI (Oryzabase and IRIS (http://www.iris.irri.org/)), and precise microscopic observation has not been done for GG chromosomes despite their markedly large DNA contents.

The accession W1213, registered as the tetraploid O. minuta (BBCC) in the NIG, is assigned as the diploid O. officinalis IRGC100973, which is one of the two duplicate stocks of W1213 at IRRI (Table 1). The chromosome constitution of W1213 preserved in NIG was $2 n=48$ (Fig. 1B). One of two duplicate stocks of the accession W1527 was registered as the tetraploid $O$. punctata IRGC105182 by IRRI. This study revealed that the W1527 preserved in the NIG had a diploid chromosome constitution as registered by the NIG (Fig. 1C). These results indicate that the species assignment of W1213 and W1527 in the NIG collection is correct at least for the plants examined in this study. On the other hand, the accession W1525 was assigned as the diploid O. eichingeri (CC) after checking the chromosome number in the NIG (Tateoka 1965), but one of two duplicates from IRRI (IRGC105181) was designated tetraploid $O$. punctata. The chromosome constitution of W1525 preserved at present in the NIG showed a tetraploid configuration of $2 n=48$ (Fig. 1D), indicating that there must have been some mistake during preservation of this accession.

The total length of chromosomes averaged from three cells were measured in an AA, two CC and two GG species (Fig. 1 and summarized in Table 3). The W1805 O. rhizomatis showed the largest DNA content and the longest chromosome length of the $\mathrm{CC}$ species investigated in this study (Fig. 1E, Table 1 and Table 3). The total chromosome length of $O$. granulata (W0003 and W0067; GG) was much longer than the other diploids (Fig. $1 \mathrm{~F}$ and Table 3 ), as expected from flow cytometry (Table 1 and Table 2). The average total length correlated significantly with average DNA content (correlation coefficient $=0.92,0.010<\mathrm{P}<0.050$ ), as Uozu et al. (1997) showed previously.

\section{Discussion}

This study revealed that accessions belonging to the same species as well as the same genome type seem to have similar genome sizes, while the genome sizes of accessions belonging to different genome types showed significant differences. However, the genome sizes of CC, CCDD and HHJJ species were diverse within the same genome type (Table 1 and Table 2). Microscopic observation also suggested structural diversity of the CC-genome chromosomes (Table 3). The diploid species that is supposed to be the DD genome donor is unidentified, though Ge et al. (1999) propose that the DD genome may be derived from $O$. australiensis, carrying the EE genome, judging from phylogenetic analysis of the $A d h 1$ and $A d h 2$ loci. Although the diversity of the DD genome size has been unclear, the diverse genome size of CCDD species may be due to diversity of CC genome size. However, in this study the DNA contents tended to be different from those reported previously for species with a large genome size. Noirot et al. (2005) propose that the genome-size estimation by flow cytometry

Table 3. Total chromosome length of a cultivated rice and wild species at early prometaphase

\begin{tabular}{lccccr}
\hline \hline Species & $\begin{array}{c}\text { Accession No. } \\
\text { or variety name }\end{array}$ & $\begin{array}{c}\text { Genome } \\
\text { type }\end{array}$ & 2n & $\begin{array}{c}\text { Chromosome }^{\mathrm{a}} \\
\text { length }(\mu \mathrm{m})\end{array}$ & S.D. $^{\mathrm{b}}$ \\
\hline O. sativa & cv. Nipponbare & AA & 24 & 86.6 & 9.9 \\
O. eichingeri & $\mathrm{W} 1527$ & $\mathrm{CC}$ & 24 & 82.8 & 14.5 \\
O. rhizomatis & $\mathrm{W} 1805$ & $\mathrm{CC}$ & 24 & 107.3 & 11.4 \\
O. granulata & $\mathrm{W} 0003$ & $\mathrm{GG}$ & 24 & 157.8 & 4.3 \\
O. granulata & $\mathrm{W} 0067$ & $\mathrm{GG}$ & 24 & 142.5 & 13.4 \\
\hline
\end{tabular}

a: Absolute chromosome lengths from 3 cells were averaged.

b: S.D. represents a standard deviation. 


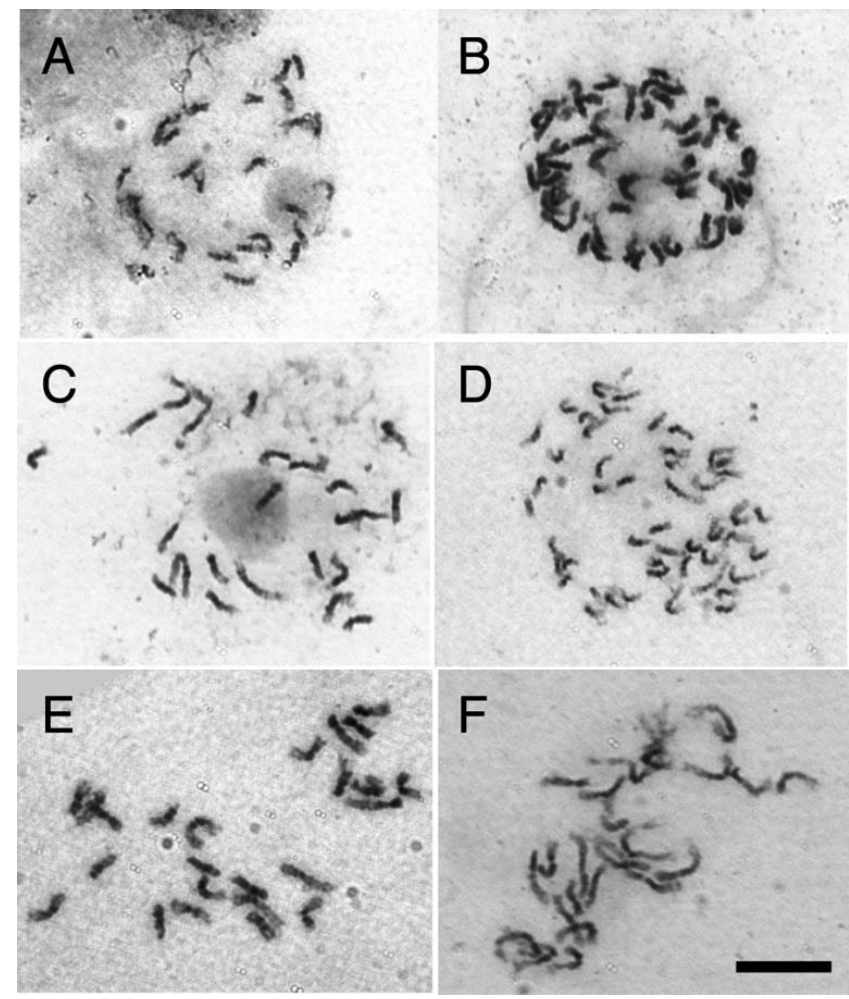

Fig. 1. Chromosome spreads of a cultivated rice (A) and wild Oryza species (B to F). Somatic cells at early prometaphase in root tip tissues were prepared. (A) O. sativa, cv. Nipponbare (AA). (B) $\mathrm{W} 1213$, O. minuta (BBCC). (C) W1527, O. eichingeri (CC), (D) W1525. This accession had been assigned as diploid $O$. eichingeri (CC), but was revealed to be a tetraploid plant with $2 \mathrm{n}=48$ chromosomes in this study. (E) W1805, O. rhizomatis (CC). (F) W0003, O. granulata (GG). Scale bar indicates $10 \mu \mathrm{m}$.

depends on the temperature condition and cytosolic compounds. For precise estimation and comparison of DNA contents, flow-cytometric procedures should be investigated thoroughly and adjusted to Oryza cells.

$O$. granulata and $O$. meyeriana, both carrying the GG genome, are genetically very close, and it is difficult to distinguish these species based on morphology except for spikelet size. Both species are the only wild Oryza species that are found in upland habitats not in or near water (Vaughan and Morishima 2003). This study revealed that both species possess a larger genome size than other diploid species (Table 1 and Table 2). This was also supported by microscopic chromosome analysis (Table 3) and corresponded well to the results shown by Ammiraju et al. (2006). In addition, O. rhizomatis also showed the largest genome size among diploids in the officinalis complex investigated in this study, though only one $O$. rhizomatis accession was examined (Table 1).

The reason for the variation in genome size among diploid Oryza species may be argued in relation to chromosomal constitution at the molecular level. One factor that has led to the EE genome becoming so large as compared to other diploids is accumulation of more than 90,000 copies of retrotransposon copies (Noma et al. 1997, Piegu et al. 2006). Recently, Lee et al. (2005) revealed that $O$. rhizomatis (CC) and $O$. brachyantha $(\mathrm{FF})$ carry specific centromere repeats, CentO-C and CentO-F, but completely lack general CentO repeats. A different constitution of CentO-C and TrsC satellites was also observed between $O$. officinalis and O. rhizomatis (Bao et al. 2006). Thus the composition of retrotransposon families and centromeric repeats should be investigated further in the CC, FF and GG genomes.

The NIG Oryza collection now has 1,725 accessions of wild species, and 282 accessions were selected as a core collection. It is difficult, however, to maintain a large number of accessions for a long time without error such as mislabeling or unconscious replacement. In this study, several mistakes were detected through assessment of ploidy level by flow cytometry and microscopic observation. For example, the W1525 conserved at present in the NIG, previously assigned as the diploid $O$. eichingeri (CC), showed a chromosome configuration of tetraploid in this study (Fig. 1C). Tateoka (1965) classified many wild relatives from Africa into two species, O. eichingeri $(2 \mathrm{n}=24)$ and $O$. punctata $(2 \mathrm{n}$ $=24$ or 48 ) according to their morphological characters and chromosome number. However, he designated W1525 and W1527 as intermediates of the two species and suggested a complexity of CC genome species. Wild species conserve their heterogeneity in natural populations to adapt to various environments. In some cases, the accession numbers may have been given to a seed mixture from multiple plants of a natural wild population, instead of a single wild plant. In addition, a complex composition of CC species was proposed by Tateoka (1965) and confirmed by this study. Therefore, the inconsistent ploidy levels within accessions such as W1525 are difficult to attribute simply to an error during seed stock processes. These accessions must be inspected for the genetic diversity of their seed stocks and in some cases, replaced by other reliable accessions.

Several core collections of AA-genome rice species have been developed based on molecular markers such as isozymes and DNA markers (Vaughan 1991, Li et al. 2004, Kojima et al. 2005). Further inspection and rearrangement of the NIG collection by genome-specific or species-specific molecular markers will also be required in addition to the flow cytometric and chromosome analyses examined in this study. Recently, a short interspersed retrotransposon element, $p$-SINE1, and its family members were used as efficient DNA markers to clarify the phylogenetic relationship among several AA genome species, all of which were derived from the NIG collection (Xu et al. 2005). Use of additional $p$-SINE members and other DNA markers for inspection of accessions other than AA genome species is now proceeding. Such analysis will help in establishing the molecular basis for phylogenetic studies and in developing a tool for using wild relatives of Oryza in alien introgression. 


\section{Acknowledgements}

This work was funded by grants from the National Bioresource Project of the Ministry of Education, Culture, Sports, Science and Technology (MEXT), Japan.

\section{Literature Cited}

Abramoff,M.D., P.J.Magelhaes and S.J.Ram (2004) Image processing with Image J. Biophotonics International 11: 36-42.

Ammiraju,J.S., M.Luo, J.L.Goicoechea, W.Wang, D. Kudrna et al. (2006) The Oryza bacterial artificial chromosome library resource: construction and analysis of 12 deep-coverage largeinsert BAC libraries that represent the 10 genome types of the genus Oryza. Genome Res. 16: 140-147.

Bao, W., W.Zhang, Q.Yang, Y.Zhang, B.Han, M.Gu, Y.Xue and Z.Cheng (2006) Diversity of centromeric repeats in two closely related wild rice species, Oryza officinalis and Oryza rhizomatis. Mol. Genet. Genomics (on line): 1-10 (DOI 10.1007).

Brar,D.S. and G.S.Khush (1997) Alien introgression in rice. Plant Mol. Biol. 35: 35-47.

Frankel,O.H (1984) Genetic perspectives of germplasm conservation. In "Genetic manipulation: impact on man and society" Arber, W.K., K.L.Llimensee, W.J.Peacock and P.Stralinger (eds.), Cambridge University Press, Cambridge. p. 161-170.

Galbraith,D.W., K.R.Harkins, J.M.Maddox, N.M.Ayres, D.P.Sharma and E.Firoozabady (1983) Rapid flow cytometric analysis of the cell cycle in intact plant tissues. Science 220: 1049-1051.

Ge,S., T.Sang, B.R.Lu and D.Y.Hong (1999) Phylogeny of rice genomes with emphasis on origins of allotetraploid species. Proc. Natl. Acad. Sci. USA 96: 14400-14405.

Jena,K.K., J.U.Jeung, J.H.Lee, H.C.Choi and D.S.Brar (2006) Highresolution mapping of a new brown planthopper $(\mathrm{BPH})$ resistance gene, $B p h 18(\mathrm{t})$, and marker-assisted selection for $\mathrm{BPH}$ resistance in rice (Oryza sativa L.). Theor. Appl. Genet. 112: 288-297.

Khush,G.S. (1997) Origin, dispersal, cultivation and variation of rice. Plant Mol. Biol. 35: 25-34.

Kojima,Y., K.Ebana, S.Fukuoka, T.Nagamine and M.Kawase (2005) Development of an RFLP-based rice diversity research set of germplasm. Breed. Sci. 55: 431-440.

Kurata,N., T.Omura and N.Iwata (1981) Studies on centromere, chromomere and nucleolus in pachytene nuclei of rice, Oryza sativa, microsporocytes. Cytologia 46: 791-800.

Kurata,N., K.Miyoshi, K.I.Nonomura, Y.Yamazaki and Y.Ito (2005) Rice mutants and genes related to organ development, morphogenesis and physiological traits. Plant Cell Physiol. 46: 48-62.
Lee,H.R., W.Zhang, T.Langdon, W.Jin, H.Yan, Z.Cheng and J.Jiang (2005) Chromatin immunoprecipitation cloning reveals rapid evolutionary patterns of centromeric DNA in Oryza species. Proc. Natl. Acad. Sci. USA 102: 11793-11798.

Li,C.T., C.H.Shi, J.G.Wu, H.M.Xu, H.Z.Zhang and Y.L.Ren (2004) Methods of developing core collections based on the predicted genotypic value of rice (Oryza sativa L.). Theor. Appl. Genet. 108: 1172-1176.

Martinez,C.P., K.Arumuganathan, H.Kikuchi and E.D.Earle (1994) Nuclear DNA content of ten rice species as determined by flow cytometry. Jpn. J. Genet. 69: 513-523.

Multani,D.S., G.S.Khush, B.G.delos Reyes and D.S.Brar (2003) Alien genes introgression and development of monosomic alien addition lines from Oryza latifolia Desv. to rice, Oryza sativa L. Theor. Appl. Genet. 107: 395-405.

Noirot,M., P.Barre, C.Duperray, S.Hamon and A.deKochko (2005) Investigation on the causes of stoichiometric error in genome size estimation using heat experiments: consequences on data interpretation. Ann. Bot. (Lond) 95:111-118.

Noma,K., R.Nakajima, H.Ohtsubo and E.Ohtsubo (1997) RIRE1, a retrotransposon from wild rice Oryza australiensis. Genes Genet. Syst. 72: 131-140.

Piegu, B., R. Guyot, N. Picault, A. Roulin, A.Saniyal, H.Kim, K. Collura, D.S.Brar, S.Jackson, R.A.Wing and O.Panaud (2006) Doubling genome size without polyploidization: Dynamics of retrotransposition-driven genomic expansions in Oryza australiensis, a wild relative of rice. Genome Res. 16: 12621269.

Snedecor, G.W. (1956) Statistical method, fifth edition. Iowa Stage College Press, Iowa. p. 251-254.

Tateoka,T. (1965) Taxonomy and chromosome numbers of African representatives of the Oryza officinalis complex. Bot. Mag. Tokyo 78: 198-201.

Uozu,S., H.Ikehashi, N.Ohmido, H.Ohtsubo, E.Ohtsubo and K.Fukui (1997) Repetitive sequences: cause for variation in genome size and chromosome morphology in the genus Oryza. Plant Mol. Biol. 35: 791-799.

Vaughan,D.A. (1991) Choosing rice germplasm for evaluation. Euphytica 54: 147-154.

Vaughan,D.A. and H.Morishima (2003) Biosystematics of the genus Oryza. In "Rice: Origin, History, Technology, and Production" Smith, C.W. (ed.), John Wiley \& Sons, Inc., Indianapolis. p. 27-65.

Xu,J.H., N.Kurata, M.Akimoto, H.Ohtsubo and E.Ohtsubo (2005) Identification and characterization of Australian wild rice strains of Oryza meridionalis and Oryza rufipogon by SINE insertion polymorphism. Genes Genet. Syst. 80: 129-134. 